\title{
Infections caused by Mycobacterium tuberculosis in recipients of hematopoietic stem cell transplantation
}

\author{
Khalid Ahmed Al-Anazi ${ }^{1}$ *, Asma Marzouq Al-Jasser ${ }^{2}$ and Khalid Alsaleh ${ }^{1}$ \\ Section of Adult Hematology and Oncology, Department of Medicine, College of Medicine, King Khalid University Hospital, King Saud University, Riyadh, \\ Saudi Arabia \\ ${ }^{2}$ Central Regional Laboratory, Ministry of Health, Riyadh, Saudi Arabia
}

\section{Edited by:}

Partow Kebriaei, The University of Texas MD Anderson Cancer Center, USA

\section{Reviewed by:}

Partow Kebriaei, The University of Texas MD Anderson Cancer Center, USA

Amir Hamdi, The University of Texas MD Anderson Cancer Center, USA

*Correspondence:

Khalid Ahmed Al-Anazi, College of Medicine, King Khalid University Hospital, King Saud University, P.O. Box: 2925, Riyadh, Central Province, Saudi Arabia

e-mail:kaa_alanazi@yahoo.com
Mycobacterium tuberculosis (M. tuberculosis) infections are uncommon in recipients of hematopoietic stem cell transplantation. These infections are 10-40 times commoner in recipients of stem cell transplantation than in the general population but they are 10 times less in stem cell transplantation recipients compared to solid organ transplant recipients. The incidence of $M$. tuberculosis infections in recipients of allogeneic stem cell transplantation ranges between $<1$ and $16 \%$ and varies considerably according to the type of transplant and the geographical location. Approximately $80 \%$ of $M$. tuberculosis infections in stem cell transplant recipients have been reported in patients receiving allografts. Several risk factors predispose to $M$. tuberculosis infections in recipients of hematopoietic stem cell transplantation and these are related to the underlying medical condition and its treatment, the pre-transplant conditioning therapies in addition to the transplant procedure and its own complications. These infections can develop as early as day 11 and as late as day 3337 post-transplant. The course may become rapidly progressive and the patient may develop life-threatening complications. The diagnosis of $M$. tuberculosis infections in stem cell transplant recipients is usually made on clinical grounds, cultures obtained from clinical specimens, tissues biopsies in addition to serology and molecular tests. Unfortunately, a definitive diagnosis of $M$. tuberculosis infections in these patients may occasionally be difficult to be established. However, M. tuberculosis infections in transplant recipients usually respond well to treatment with anti-tuberculosis agents provided the diagnosis is made early. A high index of suspicion should be maintained in recipients of stem cell transplantation living in endemic areas and presenting with compatible clinical and radiological manifestations. High mortality rates are associated with infections caused by multidrug-resistant strains, miliary or disseminated infections, and delayed initiation of therapy. In recipients of hematopoietic stem cell transplantation, isoniazid prophylaxis has specific indications and bacillus Calmette-Guerin vaccination is contraindicated as it may lead to disseminated infection. The finding that $M$. tuberculosis may maintain long-term intracellular viability in human bone marrow-derived mesenchymal stem cells complicates the development of effective vaccines and strategies to eliminate tuberculosis. However, the introduction of linezolid, cellular immunotherapy, and immunomodulation in addition to autologous mesenchymal stem cell transplantation will ultimately have a positive impact on the overall management of infections caused by $M$. tuberculosis.

Keywords: Mycobacterium tuberculosis, hematological malignancy, latency, hematopoietic stem cell transplantation, drug resistance

\section{INTRODUCTION}

Mycobacterium tuberculosis (M. tuberculosis) is an aerobic, acid fast, non-spore forming non-motile bacillus that belongs to the family Tuberculosisceae $(1,2)$. M. tuberculosis is pathogenic for humans while Mycobacterium bovis is usually an animal pathogen $(1,2)$. Tuberculosis (TB) is caused by members of M. tuberculosis complex that include: . tuberculosis, M. bovis, M. bovis BCG, $M$. africanum, M. microti, M. pinipedii, M. caprae, and M. mungi $(1,3)$.

Once infected, active TB disease develops in $10 \%$ of patients, while the remaining individuals enter into latency phase that can reactivate at a later time especially if the immunity of the individual declines $(1,4)$. Active TB develops in approximately $59 \%$ of patients and is predominantly pulmonary in nature. Extrapulmonary $\mathrm{TB}$ occurs in $41 \%$ of patients and the clinical manifestations depend on the primary site of involvement. Latent TB infection (LTBI) is not contagious, has no clinical manifestations but can reactivate following reduction in immunity $(1,4$, 5). Immunocompromised individuals including cancer patients, transplant recipients, and those receiving immunosuppressive therapies including monoclonal antibodies should be evaluated 
regularly and treated for LTBI at the time of diagnosis or just before starting immunosuppressive treatment $(1,6)$.

\section{TUBERCULOSIS INFECTIONS IN GENERAL}

Several risk factors predispose individuals to M. tuberculosis infections in the general population and these are included in Table 1 $(1,2,5,7)$. Patients with hematologic malignancies (HM) are at risk of developing $M$. tuberculosis infections. Specific predisposing factors for $M$. tuberculosis infections in this group of patients are shown in Table $2(1,5,7)$.

\section{THE GLOBAL SCENE OF M. TUBERCULOSIS INFECTIONS}

Approximately eight million new cases of TB infection are reported annually with the vast majority occurring in developing countries and most of the new cases arise as reactivations of old TB infections. Out of these eight million cases, five million patients receive some treatment and only half a million patients receive short

\section{Table 1 | Risk factors for M. tuberculosis infections in the general population}

(1) Human immunodeficiency virus infection

(2) Diabetes mellitus

(3) Hematologic malignancy and solid tumors

(4) Solid organ and hematopoietic stem cell transplantation

(5) End-stage renal disease

(6) Chronic liver disease

(7) Collagen vascular and autoimmune disorders

(8) Chronic and industrial lung diseases such as silicosis

(9) Tobacco smoking

(10) Alcoholism

(11) Use of illicit drugs

(12) Climate and travel

(13) Malnutrition

(14) Pregnancy

(15) Old age

(16) Imprisonment

(17) Genetic predisposition to $M$. tuberculosis infection

(18) Recent exposure to a patient with contagious tuberculosis

M. tuberculosis, Mycobacterium tuberculosis.

Table 2 | Risk factors for $M$. tuberculosis infections in patients with HM.

(1) The primary hematological disorder, particularly at the time of relapse

(2) Corticosteroid therapy

(3) Cytotoxic chemotherapy

(4) Radiotherapy

(5) Other immunosuppressive therapies:

- Monoclonal antibodies such as rituximab and alemtuzumab

- Tyrosine kinase inhibitors such as imatinib

(6) Old age

(7) Presence of other comorbid medical conditions such as diabetes mellitus and malnutrition

M. tuberculosis, Mycobacterium tuberculosis; HM, hematologic malignancy. courses of direct observed therapy. Recently, the global rates of TB are rising in Asia, Africa, and Latin America where co-infection with human immunodeficiency virus (HIV) is common $(1,8)$.

The world health organization (WHO) declared TB a global health emergency in the year 1993. One third of the world population has LTBI and $5-10 \%$ of latent forms become active at any time. Also, approximately $95 \%$ of TB cases and $98 \%$ of deaths related to TB infection occur in poor countries $(1,9)$. Another problem, which makes management of $M$. tuberculosis infections a difficult task, is the evolution of drug resistance. Resistance to anti-TB chemotherapy can be attributed to: (1) failure to complete the course of anti-TB treatment, (2) weak health-care infrastructures particularly in third world countries, (3) lack of diagnostic techniques and drug susceptibility testing (DST), and (4) having no new anti-TB drugs available since the 1960s till the turn of the century $(1,9)$. In a survey performed by the WHO in the year 1997, drug resistant $\mathrm{TB}$ was found in all 35 countries included in that survey and in the last survey done in the year 2000, drug resistant TB was documented in all 100 countries surveyed $(1,10)$.

\section{TUBERCULOSIS INFECTIONS IN RECIPIENTS OF HSCT}

Hematopoietic stem cell transplantation (HSCT) can be defined as the transfer of HSCs from one individual to another (allogeneic) or the return of previously harvested HSCs to the same individual (autologous) after manipulation of the cells and/or the recipient (11). Despite the recent advances in managing HSCT patients and the progress in supportive care, infection is still reported as the primary cause of death in $8 \%$ of autologous HSCT patients and $17-20 \%$ of allogeneic HSCT recipients (11). Recipients of allogeneic HSCT have severely impaired cell-mediated immunity as a consequence of: (1) pre-transplant conditioning therapies, (2) immunosuppressive treatments given in the post-transplant period, and (3) graft versus host disease (GVHD) and its treatment, which further suppress the immunity in HSCT recipients. Severe suppression of cellular immunity makes HSCT patients at risk of viral, bacterial, fungal, and mycobacterial infections $(12,13)$. Recipients of T-cell depleted allografts and those having GVHD may have more extensive and prolonged T-cell immunodeficiency and this consequently predisposes them to severe infections (14). For example, in recipients of T-cell depleted allografts in the United States of America (USA), active TB has been reported in $0.69 \%$ of patients and all the reported cases originally came from areas that are endemic for TB (14). At least $25 \%$ of $M$. tuberculosis infections in recipients of HSCT result from reactivation of LTBIs $(1,15,16)$.

\section{RISK FACTORS FOR M. TUBERCULOSIS INFECTIONS IN HSCT RECIPIENTS}

There are several risk factors for the development of M. tuberculosis infections in recipients of HSCT and these are listed in Table 3 $(1,5,13,14,17-27)$.

\section{EPIDEMIOLOGY OF M. TUBERCULOSIS INFECTIONS IN RECIPIENTS OF HSCT}

Infections caused by $M$. tuberculosis are 10-40 times commoner in recipients of HSCT than in the general population. The incidence of $M$. tuberculosis infections in recipients of HSCT varies 
Table 3 | Risk factors for $M$. tuberculosis infections in recipients of HSCT.

(1) The primary hematological disorder, particularly:

- Acute myeloid leukemia

- Chronic myeloid leukemia

- Myelodysplastic syndrome

(2) Certain conditioning therapies, particularly:

- Busulphan

- Cyclophosphamide

- Total body irradiation

(3) Corticosteroid therapy

(4) T-cell depletion in allografts

(5) Matched unrelated allogeneic HSCT

(6) Mismatched allografts

(7) Acute and chronic graft versus host disease

(8) Bronchiolitis obliterans

(9) History of M. tuberculosis infection

M. tuberculosis, Mycobacterium tuberculosis; HSCT, hematopoietic stem cell transplantation.

considerably according to the type of HSCT and to the geographical location $(1,14,21,23,26-30)$. The prevalence of $M$. tuberculosis infections is higher in recipients of solid organ transplant (SOT) than in HSCT presumably due to the longer immunosuppression in SOT recipients $(15,28,31,32)$. Incidences of $M$. tuberculosis infections in HSCT recipients have been reported to be as low as $0.0014 \%$ in the USA and as high as $16 \%$ in Pakistan (33). In countries, such as Spain and Turkey, the incidence M. tuberculosis was $1.6 \%$ while in Taiwan and Hong Kong, it was $8.57 \%$. The vast majority of $M$. tuberculosis infections have been reported from Asia and the incidence of TB in recipients of allogeneic HSCT is directly proportional to the incidence of $\mathrm{TB}$ in the general population $(1,14,21,23,26-29)$. Approximately $95 \%$ of TB infections in allogeneic HSCT patients have been reported from developing countries, where HSCT is infrequently performed, while the incidence of $M$. tuberculosis infections in allograft recipients is low in developed countries where HSCT is often performed. Usually, M. tuberculosis infections develop 45-365 days post-HSCT and most of the cases reported occurred after 90 days of HSCT. Studies have shown that the diagnosis of TB infections is usually made at a median of 257.2 days after HSCT and the range varies from as early as 21 days to as late as 1410 days post-HSCT $(1,19,21,22,32,33)$.

\section{CLINICAL ASPECTS OF M. TUBERCULOSIS INFECTIONS IN HSCT RECIPIENTS}

In recipients of HSCT, the lung is the most commonly involved organ and lung involvement has been reported in $50-100 \%$ of allografts $(1,21,33-36)$. Pulmonary tuberculous infections after HSCT are 13.1-fold higher than in the general population. Also, the incidence of pulmonary TB infections is higher in recipients of allogeneic HSCT than in recipients of auto-grafts. Pulmonary TB may coexist with invasive fungal infections such as mucormycosis or aspergillosis in HSCT recipients $(1,21,33-36)$. In these patients, lung involvement by TB may resemble that of invasive fungal pulmonary infections and patients may even present with bacteremia caused by $M$. tuberculosis and a rapidly progressive illness. In such situations, the use of computed axial tomography (CAT scans) and molecular techniques such as polymerase chain reaction (PCR) may accelerate the diagnosis in this group of immunocompromised hosts (34). Pulmonary TB should be considered in the differential diagnosis of lung infections in HSCT recipients living in geographical locations that are endemic for TB (37).

At least one third of M. tuberculosis infections in recipients of HSCT are disseminated at presentation with predominant extra-pulmonary involvement. Extra-pulmonary sites of infection include: liver, spleen, bone, bone marrow, brain, and spine $(1,13,16,24,32,38,39)$. Central nervous system involvement may take the form of space occupying lesions in the brain. Abdominal involvement may be in the form of acute abdomen due to abdominal masses causing acute abdominal pain and intestinal obstruction $(1,13,16,24,32,38,39)$. In recipients of allogeneic HSCT, M. tuberculosis infections may present in an atypical manner such as pyrexia of unknown origin, non-specific features particularly in patients with extra-pulmonary involvement. Therefore, it is essential not only to have a high index of suspicion but also to apply prompt and appropriate diagnostic tools as well $(13,40)$.

The approach to LTBIs in recipients of allogeneic HSCT varies considerably from one transplant center to another (14). The diagnosis of LTBI is also difficult in immunocompromised individuals as they are likely to have attenuated response to tuberculin skin test (TST). To prevent reactivation of LTBI in recipients of allogeneic HSCT, it is recommended to consider administration of isoniazid (INH) prior to and post-HSCT particularly in patients living in areas that are endemic for TB (14).

\section{TUBERCULOSIS INFECTIONS IN RECIPIENTS OF CERTAIN TYPES OF HSCT}

M. tuberculosis infections have been reported in various forms of $\operatorname{HSCT}(5,16,19,26,29,32,41-44)$. The exact incidence of $M$. tuberculosis infections among recipients of umbilical cord blood transplant (UCBT) is unknown $(16,26,41)$. The interval between UCBT and the diagnosis of TB infection varies considerably from as early as 21 days to as late as 3 years post-transplant. Also, M. tuberculosis infections in recipients of UCBT may have a rapidly progressive course and present with bacteremia or disseminated infection $(16,26,41)$. Infections caused by M. tuberculosis have been reported in patients receiving autologous HSCT and the incidence varies from 0.0 to $0.23 \%(5,19,32,42)$. Old literature suggested that the risk of $M$. tuberculosis infections in recipients of autologous HSCT was similar to that in the general population and that no obvious risk factors were associated with the development of TB infections in autograft recipients $(19,29)$. However, over the last decade, M. tuberculosis infections have been increasingly reported in recipients of autologous HSCT $(43,44)$. Also, such infections have been reported as early as day 30 post-autologous HSCT and M. tuberculosis infections in these patients have been reported to coexist with other infections such as cytomegalovirus infection and adenovirus-related hemorrhagic cystitis. M. tuberculosis infections in autograft recipients may present with pulmonary involvement as well as extra-pulmonary manifestations such as bone marrow involvement with pancytopenia and myeloid 
maturation arrest $(43,44)$. The recent use of monoclonal antibodies, such as rituximab, to control the primary underlying disease could have contributed to the recent increase in the incidence of $M$. tuberculosis infections in recipients of autologous HSCT $(43,44)$.

\section{DIAGNOSIS OF M. TUBERCULOSIS INFECTIONS IN RECIPIENTS OF HSCT}

The diagnosis of M. tuberculosis infections in HSCT recipients should be based on: clinical grounds, sputum microscopy and cultures, cultures of pleural and pericardial fluid in addition to bronchoalveolar lavage (BAL) samples, bone marrow cultures, serology, molecular testing, and tissue biopsies (1,40). Tissue biopsies from liver, spleen, lungs, lymph nodes, and bone marrow may show caseating or non-caseating granulomas $(1,40)$. The histology may be atypical showing either granuloma in the absence of caseation necrosis or lack of granuloma in the presence of caseous necrosis. The latter can be explained by impaired T-cell function in the early post-HSCT phase $(1,16,26)$. Unfortunately, a definitive diagnosis of M. tuberculosis infections in HSCT recipients is usually difficult to be established because: immunological defecits may lead to mild and non-specific clinical manifestations and histology does not usually show typical granuloma formation $(1,16$, 26). Therefore, a high index of suspicion should be maintained in recipients of HSCT living in endemic areas and presenting with: unexplained fever, cough, pleuritic chest pain, diffuse reticulonodular shadows on chest X-ray, rapidly progressive illness, and disseminated infection $(1,12,20,32,37,40)$.

\section{TREATMENT OF M. TUBERCULOSIS INFECTIONS IN HSCT RECIPIENTS}

Provided there is no evidence of drug resistant strains of $M$. tuberculosis, the recommended treatment schedule for adults with $M$. tuberculosis infections includes: (1) an induction phase for 2 months composed of the standard first-line anti-TB agents: rifampicin: $600 \mathrm{mg} /$ day, $\mathrm{INH}: 300 \mathrm{mg} /$ day, pyrazinamide $1600 \mathrm{mg} /$ day, and ethambutol $1200 \mathrm{mg} /$ day, and (2) a maintenance phase for 7-10 months with rifampicin $600 \mathrm{mg} /$ day and INH $400 \mathrm{mg}$ /day (21). Multidrug-resistant TB (MDR-TB) has been reported in recipients of allogeneic HSCT. MDR-TB is more frequent in SOT recipients than in HSCT patients (45). The potential risk of MDR-TB may act as a major obstacle to effective treatment of M. tuberculosis infections following HSCT despite appropriate anti-TB medications (46). Early introduction of second-line anti-TB chemotherapy may have a good outcome (45).

\section{CHEMOPROPHYLAXIS IN RECIPIENTS OF HSCT}

Isoniazid prophylaxis has been successfully used to prevent reactivation of old TB infections in recipients of HSCT. However, routine prophylaxis against $\mathrm{TB}$ infections in patients with skin test reactivity and a normal chest $\mathrm{X}$-ray should be balanced against the possibility of drug-induced hepatotoxicity in recipients of HSCT $(1,18,23,29,35,37,42,47)$. Therefore, INH prophylaxis should not be given routinely, but close follow-up and monitoring for reactivation of latent infections is recommended. In geographical locations where TB is prevalent: pre-and post-HSCT follow-up for TB should be taken into consideration, and the use of INH prophylaxis should be seriously considered (1, 18, 23, 29, 35, 37, 42, 47).
Indications for INH prophylaxis in candidates and recipients of HSCT include: (1) exposure to an individual with active, infectious (sputum-smear positive) pulmonary, or laryngeal TB regardless the TST or interferon-Gamma release assay (IGRA), (2) Having a positive TST results, regardless of prior bacillus Calmettte-Guerin (BCG) vaccination without previous treatment and no evidence of active TB disease, and (3) having a positive IGRA result, without previous anti-TB therapy and no evidence of active TB disease $(11,15,35)$. Exposure of a candidate or a recipient of HSCT to an active, but non-infectious, patient with extra-pulmonary $\mathrm{TB}$ does not require preventive therapy. The value of INH prophylaxis in recipients of HSCT living in countries with high prevalence of TB should be considered at institutional or regional levels. In countries with high prevalence of MDR-TB, single-agent prophylaxis may be ineffective $(11,15,35)$. In recipients of HSCT living in countries that are endemic for $\mathrm{TB}, \mathrm{INH}$ prophylaxis has been shown to be effective in preventing TB in these immunocompromised hosts $(5,28,48)$. It is recommended that INH should be administered for at least 6-9 months and that it should be commenced prior to or immediately after completion of conditioning therapy for HSCT $(11,28,48)$. INH prophylaxis is well tolerated post-HSCT even with concurrent administration of fluconazole. However, concurrent use of INH with itraconazole is not recommended. The impact of using voriconazole or posaconazole on INH prophylaxis is still unknown (11).

Other prophylactic regimens include rifampicin for 4 months although its use is associated with substantial drug interactions with immunosuppressive agents and other medications. Rifampicin and pyrazinamide combination is not recommended because of significant hepatotoxicity that can be caused by this drug combination (11). BCG vaccination is contraindicated in recipients of HSCT due to the risk of having disseminated BCG infection. Donors who live or originate from countries that are endemic for TB have a risk of active TB infection or LTBI similar to the rest of the population. These donors need to be assessed thoroughly and active TB should be ruled out in them prior to donation of HSCs (11).

\section{COURSE AND PROGNOSIS OF M. TUBERCULOSIS INFECTIONS IN HSCT RECIPIENTS}

In recipients of HSCT having $M$. tuberculosis infections, high mortality rates are encountered in patients with miliary TB and disseminated TB infections $(1,16,19,21,33,36)$. Mortality rates are higher in allogeneic HSCT than in autologous HSCT recipients. Mortality rates due to $M$. tuberculosis infections in recipients of HSCT range from 0.0 to $75 \%$ and mortality is related to the type of HSCT, the degree of immunosuppression, and depends on how early the diagnosis of $M$. tuberculosis infection is made $(1,16$, 19, 21, 33, 36).

Usually, M. tuberculosis infections in recipients of HSCT are localized to a certain organ such as lungs or central nervous system $(1,16,30,31)$. Occasionally, the infection is disseminated and the course may be rapidly progressive and the following complications may be encountered: disseminated infection, severe hyperpyrexia, adult respiratory distress syndrome, hypotension, hypoxia, sepsis, multi-organ failure, and death. However, early diagnosis, prompt initiation of appropriate anti-TB chemotherapy and having a 
localized infection as well as a drug-sensitive strain carry good outcome $(1,16,30,31)$.

\section{NEW INSIGHTS IN THE PATHOGENESIS, DIAGNOSIS, AND MANAGEMENT OF TB RECENT FINDINGS IN THE IMMUNOPATHOGENESIS OF TB GRANULOMAS}

The immunology of TB is complex and multifaceted. Identifying the immune mechanisms that lead to control of initial infection and prevent reactivation of LTBI is crucial to combat TB (49). One of the main features of the immune response to $M$. tuberculosis is the formation of granulomas (50). Understanding granulomas requires an analysis of the complex interplay of innate and adaptive molecular signals that control the focal accumulation of inflammatory cells and activity of their cellular components (51). Granulomas consist of macrophages that produce cytokines such as interferon- $\gamma$, highly differentiated cells such as multinucleated giant cells, epithelial cells, and foamy cells, surrounded by a rim of lymphocytes that include CD4 T-cells $(50,52,53)$. Despite that granulomas act to constrain the infection, some bacilli can actually survive inside them in a dormant state for a long time (50, 52-54). Although granuloma formation seems to be primarily a host defense mechanism for containing bacteria, it also shelters bacteria by providing them with a niche in which they can persist in a latent form until an opportunity arises for reactivation and spread. Also, evolution of drug resistance develops during the latency phase (50, 52-54).

For unclear reasons, acid fast bacilli (AFB) will reactivate in $10 \%$ of the latently infected individuals, escape the granuloma and spread throughout the body, thus giving rise to clinical disease, and are finally disseminated throughout the environment (50). Studies in mice have shown that production of pro-inflammatory cytokines, growth factors, and leukocyte surface markers by granuloma cells indicates continued processes of activation and deactivation of granuloma inflammatory cells during the progress of LTBI (52).

Understanding the pathophysiology of granulomas is critical for the design of new vaccines and anti-TB drugs. Chemokine receptor analysis of granulomas has revealed an age-dependent heterogeneity of immunological responses, thus chemokines may become potential targets for therapeutic interventions $(50-52,55)$. It is unrealistic to call TB granuloma as an unsuccessful host defense as it successfully contains the infectious focus in more than $90 \%$ of cases. The $10 \%$ of individuals who progress to active TB suffer from disturbances in the balance between inflammatory reaction to the infectious agent and immunological defenses of the host (54). Granulomas are protective for dormant AFB, but are capable of having tissue destructive nature by behaving as a tumor rather than an active site of bacterial control (55-57). Granulomas are highly dynamic and are shaped by the pathogen and the immune response elements of the host. To secure transmission to a new host, $M$. tuberculosis has evolved to drive T-cell immunity to the point that necrotizing granulomas leak into the bronchial cavities to facilitate expectoration of AFBs. Complete eradication of AFB in granulomas does not occur since $M$. tuberculosis is able to persist within the granuloma, reactivate, and escape under certain circumstances (57).

\section{ROLE OF MESENCHYMAL STEM CELLS IN THE PATHOGENESIS OF TB}

Despite the generation of robust host immune responses, $M$. tuberculosis can successfully evade host immunity to establish a persistent infection by incompletely understood mechanisms. M. tuberculosis suppresses T-lymphocyte responses by recruiting mesenchymal stem cells (MSCs) into the site of infection (58). Recruitment of MSCs to the periphery of granulomas plays a crucial role in the pathogenesis of $M$. tuberculosis infection by confining the organism within the granulomas on one hand and keeping M-TB-specific T-cells at the bay on the other hand (58). Thus, large numbers of MSCs infiltrate into the site of TB infection and position themselves between the harbored pathogen and effector T-cells that target the pathogen. MSCs suppress Tlymphocyte responses by producing nitrous oxide. Therefore, targeting MSCs or nitrous oxide seems a feasible therapeutic intervention for designing new effective therapeutic and preventive strategies against TB (58-60).

M. tuberculosis may maintain long-term intracellular viability in a human bone marrow-derived CD271 $/$ CD45-(BM-MSC) population in vitro. Also, M. tuberculosis resides in an equivalent population of BM-MSCs in a mouse model of dormant TB infection (61). Viable M. tuberculosis has been detected in $\mathrm{CD} 271^{+} / \mathrm{CD} 45^{-}$BM-MSCs isolated from individuals who had successfully completed months of anti-TB drug therapy. Thus, $\mathrm{CD}_{271^{+}}$BM-MSCS may provide a long-term protective intracellular niche in the host in which dormant $M$. tuberculosis can reside. M. tuberculosis can persist in hostile intra-cellular microenvironments evading immune cells and drug treatment. Finally, BM cellular niche may be important for the maintenance of the nonreplicating phase of the M. tuberculosis life cycle (61). Glycation of the functional domain of $\mathrm{CD} 271^{+}$MSCs, which have been proven to be the protective niche for M. tuberculosis, occurs in patients with uncontrolled DM and can modulate the genesis of LTBIs in chronic DM (62).

\section{NEW DIAGNOSTIC TOOLS FOR TUBERCULOSIS}

The most common direct method for diagnosing TB worldwide is sputum-smear microscopy, which was developed more than 100 years ago, where bacteria are observed in sputum samples examined under a microscope (63). Acid resistance is one of the main features of mycobacteria that allows for quick identification. Consequently, sputum culture remains the gold standard for the diagnosis of pulmonary TB (63). Liquid media are considered the standard method for the isolation and culture of M. tuberculosis as they have a better quality of isolation compared to solid media. The introduction of liquid culture-based techniques was a great improvement for diagnosis, shortening the time to detection to $10-14$ days instead of several weeks needed for conventional media (63).

Molecular detection of M. tuberculosis continues to change the landscape of TB diagnosis. Because of the slow growth rate of M. tuberculosis, conventional methods for its detection based on solid culture media take several weeks to yield results (63). With the purpose of obtaining faster results and earlier diagnosis of M. tuberculosis infection, several molecular methods have been introduced and evaluated in numerous studies. These molecular tests are summarized in Table 4 (63-68). M. tuberculosis-specific 
Table 4 | It shows new diagnostic tests for $M$. tuberculosis.

\begin{tabular}{lc}
\hline Category & Type of test/technology used \\
\hline Immunodiagnostics & (1) $\gamma$ interferon release assays; IGRAs for \\
diagnosis of latent TB infection \\
(2) Tuberculosis biomarkers
\end{tabular}

\section{Examples}

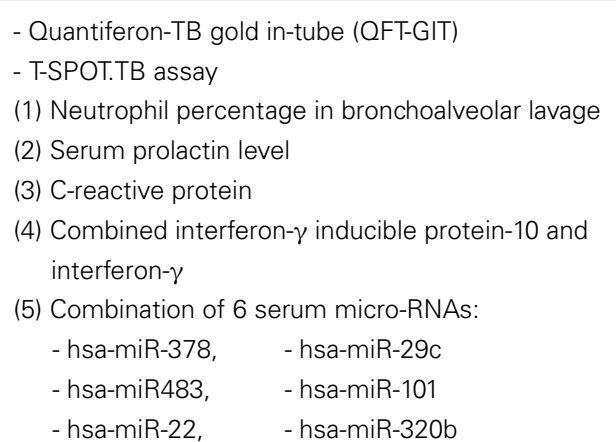

Molecular tests used for detection of $M$. tuberculosis in clinical specimens

Detection of drug resistance

New technologies in the pipeline for detection of $M$. tuberculosis
(1) Nucleic acid amplification assays

(2) Lateral flow assays

(3) Line probe assays

(4) DNA sequencing techniques
- Accu Probe (Gen-Probe, Inc., San Diego, CA, USA)

- INNO-LiPA Mycobacteria (LiPA, Innogenetics, Ghent, Belgium)

- Geno Type Mycobacterium assay (Hain Diagnosika, Germany)

-TB peptide nucleic acid fluorescence in situ hybridization (Dako, A/S Glostrup, Denmark)

- PCR-restriction fragment length polymorphism analysis (PRA)

- DNA microarray or high density oligonucleotide arrays

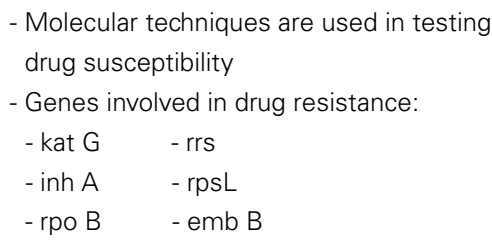

(1) Line probe assays: PCR-based reverse-hybridization line probe assay (Inno-LiPA Rif TB test, Innogenetics, NV, Ghent, Belgium)

(2) Real-time PCR Xpert MTB assay for detection of rifampicin resistant $M$. tuberculosis strains

(3) Array-based technologies: multiplex PCR for detection of genetic mutations involved in drug resistance

(1) LAM-urine antigen detection using ELISA techniques

(2) Use of chromatographic techniques for identification of volatile organic compound as markers in clinical specimens

(3) Bead-based methods for detection and identification

(4) Simplified smart flow cytometry

(5) Broad nucleic acid amplification-mass spectrometry

M. tuberculosis, Mycobacterium tuberculosis; PCR, polymerase chain reaction; ELISA, enzyme-linked immunosorbent assay.

nucleic acid amplification tests (NAATs) are the most frequently used molecular tests for laboratory diagnosis of TB (63). The NAAT tests provide a reliable way of increasing the specificity of diagnosis but sensitivity is too poor to rule out disease particularly in smear-negative disease where clinical diagnosis is equivocal and where the need is greatest (4). For pleural TB and TB meningitis, adenosine deaminase (ADA) tests have high sensitivity but limited specificity. NAATs have high specificity to rule in disease and could be used in conjunction with ADA and interferon- $\gamma$ to increase sensitivity for ruling out disease (4).

Critical parameters of newly developed enzyme-linked immunosorbent assay (ELISA) have been optimized and the cocktail antigens have specificity and sensitivity of $>98 \%$ as compared to other commercially available diagnostic tests. The newly developed ELISA that employs a cocktail of secretary proteins of $M$. tuberculosis, is an effective tool that can be used for routine screening and early-stage diagnosis of active TB (69).

Automated liquid culture systems and molecular line probe assays are recommended by the WHO as the gold standard firstline DST. The use of this system has not been cleared by the food and drug administration (FDA) in the USA for DST of secondline drugs and thus most laboratories rely on agar proportion as the reference standard (63). With the purpose of detecting drug resistance in a shorter time, several molecular assays have been introduced and these are listed in Table 4 (63-68). Studies have shown that genetic mutations play a significant role in the evolution of drug resistance. Examples of the genes involved in drug resistance are shown in Table 4 (68). Also, examples of the new technologies for detection of M. tuberculosis that are in the pipeline are included in Table 4 (65). 


\section{TUBERCULOSIS BIOMARKERS}

Tuberculosis biomarkers have the following potential applications: (1) classifying patients at a single time point as having active TB, LTBI, or no disease, (2) predicting future risk of reactivation, (3) monitoring eradication of LTBI, and (4) predicting end points for clinical trials by serving as surrogate markers of cure following anti-TB chemotherapy or vaccination against TB. Unfortunately, the progress in developing biomarkers for TB infection, in general, has been slow (70). However, a number of biomarkers have been shown to be useful in the diagnosis of pulmonary TB in immunocompromised individuals and these are shown in Table 4 (71-74). Several studies testing new biomarkers and utilizing new technologies such as proteomics, transcriptomics and metabolomics are underway. Hopefully, these trials will ultimately determine a panel of biomarkers that can help not only in establishing the diagnosis but also in the follow-up of TB infections (70).

\section{IGRAs IN THE DIAGNOSIS OF TB}

The diagnosis of LTBI is recommended in patients with HM and in recipients of HSCT. New in vitro T-cell based IGRAs, which utilize specific $M$. tuberculosis antigens have been introduced into routine practice in recent years (75). TST is the most widely used test for the diagnosis of TB infections and it has been an important and a traditional way for detecting LTBIs for a long time. However, the operational and biological limitations of TST indicate the need for an improved approach to diagnose TB infections. An accurate diagnosis of TB infection is very essential to prevent the progress from LTBI to active TB so that the overall burden of TB disease is diminished. In patients with HM and in recipients of HSCT, T-SPOT.TB may be a more useful screening test for LTBI and active TB than TST (75). IGRAs are diagnostic tools for LTBI and they are not affected by BCG vaccination status. There are two major IGRAs available: (1) quantiferon-TB gold in-tube (QFTGIT) assay and (2) T-SPOT.TB assay $(65,75-77)$ (Table 4). IGRAs have sensitivity of more than $95 \%$ for diagnosis of LTBI. However, the sensitivity of T-SPOT.TB appears to be higher than that of QFT-GIT or TST (76). In the USA, the 2010 centers for disease control and prevention (CDC) guidelines indicate that IGRAs can be used in stead of TST in all situations in which the CDC recommends TST as an aid in diagnosing $M$. tuberculosis infections. IGRAs are preferred for patients with history of BCG vaccination while TST is preferred for testing children under the age of 5 years (76). The 2013 Canadian TB standards indicate that both TST and IGRAs are acceptable alternatives for LTBI diagnosis, either test can be used for LTBI screening in any of the situations in which testing is indicated with some preferences and exceptions. For serial testing in populations exposed to TB, data are insufficient for interpretation of IGRA conversion and reversion (76). Positive QFT.TB assay results predict development of TB in recipients of HSCT in whom LTBI cannot be detected by TST (77).

\section{THE ROLE OF RADIOLOGY IN M. TUBERCULOSIS INFECTIONS}

Pulmonary infections are an important cause of morbidity and mortality during the course of treatment in patients with HM and in recipients with HSCT (78). In these immunocompromised patients: bacterial, viral, fungal, and mycobacterial organisms may infect the lungs (78). Occasionally, the infectious agents can be recovered but, at times, cultures are negative and invasive procedures such as BAL or transbronchial biopsies are required to determine the etiology of pulmonary infiltrates. Radiological findings may be helpful in predicting the etiology of pulmonary infection and may also help in prompt initiation of appropriate antimicrobial therapy (78).

High resolution (HR) CAT scans of chest are the preferred method for evaluation of a lung infiltrate over plain chest radiography because they are more sensitive in detecting pulmonary infiltrates earlier and they are more capable of better characterization of the infiltrates (79). In patients with HM and in recipients of HSCT, HR-CAT scans have a high degree of sensitivity $(>85 \%)$, a high negative predictive value $(>85 \%)$ in detecting pneumonia and a gain of 5 days compared to chest X-rays. Consequently, clinical management is changed based on HR-CAT scan findings (79). CAT scan appearances in pulmonary TB include: air space consolidation; pulmonary nodules; interstitial or septal thickening; cavity formation; tree-in-bud sign; ground glass opacification; pleural effusions, as well as enlarged and may be necrotic lymph nodes $(78,80)$. In patients with febrile neutropenia, HR-CAT scan of chest is an excellent modality in the diagnostic work-up allowing early detection and characterization of pulmonary abnormalities. In pulmonary TB, HR-CAT scans have $90 \%$ sensitivity and $97.02 \%$ specificity (80).

\section{THE EMERGING ROLE OF PET SCANS IN TB DIAGNOSIS}

CAT scans and magnetic resonance imaging (MRI) provide excellent structural resolution for visualization of areas of infection and inflammation, but mainly after becoming so advanced to cause significant anatomical tissue damage $(81,82)$. Fluorodeoxyglucose positron emission tomography (FDG-PET) scans are highly sensitive in detecting foci of infection early and they fulfill most of the criteria established for molecular imaging. In patients with $\mathrm{TB}$ infections, FDG-PET scans can show intense multifocal uptake in involved areas, such as lymph nodes, bones, and lung parenchyma, and they determine active foci of infection as well as the extent of these infections. They are useful in monitoring and follow-up of complications related to these infections $(81,82)$.

On PET scans, TB appearances may mimic those of malignancy such as lymphoma or other infections such as invasive fungal infections (83). C-11 acetate accumulates in tumors but not in infectious or inflammatory lesions. Therefore, incorporation of C-11 acetate in PET scanning may help to differentiate TB from malignancy (83). Although a high standardized uptake value (SUV) of more than 2.5 is usually attributed to malignancy, SUV values ranging between 2 and 21 have been described in patients with tuberculous infections (83).

\section{NEW ANTI-TB THERAPIES}

Barriers to improvements in the outcomes of anti-TB therapies include: (1) long treatment duration resulting in poor patient adherence to treatment and loss of patients follow-up, (2) complex therapeutic regimens that involve expensive and toxic medications, (3) toxic effects and drug interactions particularly if the patient is receiving other medications such as antiretroviral treatment or immunosuppressive therapies following transplantation, and (4) evolution of MDR strains (84). MDR isolates of M. tuberculosis 
arise as a consequence of sequential accumulation of a genetic mutation conferring resistance to single therapeutic agents (85). MDR-TB is caused by M. tuberculosis that is resistant to at least INH and rifampicin, which are the two most effective first-line anti-TB drugs $(84,86,87)$. Extensively drug-resistant TB (XDRTB) is MDR-TB with additional resistance to any of the fluoroquinolones and to at least one of three injectable anti-TB agents such as amikacin, kanamycin, and capreomycin $(84,86,87)$.

After 50 years of no new anti-TB drug development, a promising pipeline is emerging through the repurposing of old drugs, re-engineering of existing antibacterial compounds, and discovery of new and novel therapies that are active against dormant as well as persistent populations of M. tuberculosis (84). New drugs and chemical compounds have been tried with success in the treatment of infections caused by M. tuberculosis and these are included in Table 5 (84, 88, 89). New drugs and new combination regimens in clinical trials are expected to increase therapeutic efficacy and shorten treatment duration in both drug-susceptible and drug-resistant strains of $M$. tuberculosis $(84,88,89)$. Studies in non-human primate models of active TB and LBTI have shown that targeting mycobacteria in hypoxemic environments by administration of drugs such as metronidazole may not only prevent reactivation of LTBI but may shorten the treatment duration of active TB as well (90).

The use of immunotherapy as an adjunct to anti-TB chemotherapy may improve success rates for treatment of

Table 5 | It shows new therapeutics active against $M$. tuberculosis.

\begin{tabular}{|c|c|c|}
\hline Type of therapy & \multicolumn{2}{|l|}{ Examples } \\
\hline $\begin{array}{c}\text { (1) New antimicrobials active } \\
\text { against } M \text {. tuberculosis }\end{array}$ & $\begin{array}{l}\text { - New fluoroquir } \\
\text { fluoroquinolon } \\
\text { - Levofloxacin } \\
\text { - Moxifloxacin } \\
\text { - Rifaquine } \\
\text { - Linezolid, } \\
\text { - Clofazimine, }\end{array}$ & $\begin{array}{l}\text { cones and } \\
\text { - OFLOining compounds } \\
\text { - NIRT } \\
\text { - REMoxTB } \\
\text { - Rifapentine } \\
\text { - Bedaquiline and } \\
\text { delamanid }\end{array}$ \\
\hline $\begin{array}{l}\text { (2) Chemical agents active } \\
\text { against } M \text {. tuberculosis }\end{array}$ & $\begin{array}{l}\text { - Sutezolid, } \\
\text { - AZD 5847, } \\
\text { - PA-824, } \\
\text { - TMC-207, }\end{array}$ & $\begin{array}{l}\text { - OPC-67683 } \\
\text { - SQ-109 } \\
\text { - } \mathrm{BTZ}-043 \\
\text { - PNU-100480 }\end{array}$ \\
\hline $\begin{array}{l}\text { (3) Adjunctive } \\
\text { immunotherapy }\end{array}$ & $\begin{array}{l}\bullet \text { IL-2, } \\
\text { • IL-7, }\end{array}$ & $\begin{array}{l}\text { - IL-24 } \\
\text { • Interferon- } \gamma\end{array}$ \\
\hline $\begin{array}{l}\text { (4) Autologous mesenchymal } \\
\text { stem cell transplantation }\end{array}$ & $\begin{array}{l}\text { - In conjunction } \\
\text { chemotherapy } \\
\text { - Effective again } \\
\text { strains }\end{array}$ & $\begin{array}{l}\text { jith anti-tuberculous } \\
\text { MDRTB and XDRTB }\end{array}$ \\
\hline $\begin{array}{l}\text { (5) Targeted M. tuberculosis } \\
\text { therapy }\end{array}$ & $\begin{array}{l}\text { - Animal studies } \\
\text { metronidazole } \\
\text { in hypoxemic e }\end{array}$ & $\begin{array}{l}\text { lave shown that } \\
\text { argets } M \text {. tuberculosis } \\
\text { vironment }\end{array}$ \\
\hline
\end{tabular}

M. tuberculosis, Mycobacterium tuberculosis; IL, interleukin.

MDRTB, multidrug-resistant tuberculosis; XDRTB, extensively drug resistant tuberculosis.
MDR-TB, shorten the time for drug-sensitive TB, and improve the immunity of individual by enhancing TB elimination to prevent disease recurrence. The adjunctive immunotherapies are listed in Table 5 (91).

\section{ROLE OF AUTOLOGOUS HSCT IN THE TREATMENT OF $M$. TUBERCULOSIS INFECTIONS}

Recently, autologous HSCT has successfully been used in the treatment of MDR-TB and even XDR-TB (Table 5) (92, 93). In one study, 27 patients, in whom previous long-term treatment with anti-TB drugs alone had been ineffective, were included (15 with MDR-TB and 12 with XDR-TB). All patients received autologous MSCs. Positive clinical responses were obtained in all 27 patients, bacterial discharge from lungs stopped in 20 patients after 3-4 months, and resolution of tissue damage and lung cavitation was obtained in 11 patients (92). In the 16 patients who had follow-up for 18-24 months: persistent remission of tuberculous process was achieved in nine patients and significant positive bacteriological and morphological responses were obtained in six patients. So, inclusion of transplantation of autologous MSCs into the course of anti-TB treatment may be a promising maneuver to enhance the efficacy of treatment in patients with drug-resistant pulmonary TB (92).

In another phase 1 clinical trial, 30 patients with microbiologically confirmed MDR-TB or XDR-TB were included (93). After 4 weeks of anti-TB drug therapy autologous HSCT was performed. Autologous bone marrow-derived MSCs were infused and the dose of MSCs was $1 \times 10^{6}$ cells $/ \mathrm{kg}$ body weight. The adverse events encountered were mostly grade I or II (93). Therefore, in patients with drug resistant $\mathrm{TB}$, autologous MSCs can be used in combination with standard anti-TB chemotherapy. However, adjunct therapy using MSCs needs to be evaluated in controlled phase 2 trials to assess effects on immune responses and clinical as well as microbiological outcomes of this newly evolving therapeutic modality (93).

\section{VACCINATION AGAINST M. TUBERCULOSIS INFECTIONS}

The BCG vaccine was developed as an attenuated live vaccine for TB control almost a century ago. Despite being the most widely used vaccine in human history, it has two major limitations: its poor efficacy against adult pulmonary TB and its disconcerting safety in immunocompromised hosts (94-96). During the past 5 years, an alarming increase in the number of patients with MDR$\mathrm{TB}$ and XDR-TB has been noted, particularly in East Europe, Asia, and South Africa. Treatment outcomes, with the available therapeutic regimens for DR-TB, are usually poor (97). Although substantial progress in drug development for TB has been achieved, scientific progress toward development of interventions for prevention and improvement of drug treatment outcomes have lagged behind. So, innovative interventions and novel adjunct treatments are needed to combat the growing pandemic of MDR-TB and XDR-TB by improving its cure rates (97).

A novel, safe, widely applicable and more effective vaccine against TB is desperately sought to achieve disease control. Over the last 20 years, tremendous progress has been achieved in TB vaccine research and development from a pipeline virtually empty of new TB candidate vaccines in the early 1990s to an era in which almost 20 vaccine candidates are present at different stages in the 
clinical trial pipeline $(97,98)$. The potential TB vaccines are either subunit vaccines aimed at boosting BCG-prime vaccination or recombinant $B C G$ constructs that may replace $B C G$ vaccine in the future $(95,96)$. Additional vaccine candidates will enter clinical trials in the near future including post exposure vaccines for individuals with LTBI. Ultimately, vaccines that prevent or eradicate M. tuberculosis infection would be the best possible option (95). The next 10-15 years will be critical for TB vaccines to demonstrate protective efficacy and safely. Next generation vaccines should be designed with the aim of preventing infection or achieving sterile eradication perhaps by redirecting immune responses at $M$. tuberculosis antigens expressed during latency or by using new platforms (98).

Having a strategy of host-directed therapies focused on the immune response of anti-TB treatment could be particularly beneficial for patients with MDR-TB or XDR-TB. Hence, more attention should be given to host-directed preventive and therapeutic interventions (97). Improved knowledge of immunology, molecular microbiology, cell biology, and biotechnology has paved the way toward effective and safe vaccines against TB. The pipeline of new vaccine candidates from preclinical to clinical testing could be accelerated by development of biomarkers that can predict the clinical outcome of TB (95). As there is no clear regulatory pathway for TB vaccines, global sharing of information among regulatory authorities from countries engaged in development of TB vaccines is required (99).

\section{CONCLUSION}

M. tuberculosis infections carry significant morbidity and mortality in recipients of various forms of HSCT particularly in patients living in endemic geographic locations. These infections have a number of risk factors and they cause a wide spectrum of clinical manifestations and complications. A high index of suspicion should be maintained in HSCT recipients presenting with compatible clinical or radiological features. Prompt diagnosis and early institution of appropriate therapy are associated with favorable outcome.

The plethora of new diagnostic techniques will hopefully help clinicians, radiologists, and laboratory staff to have early diagnosis. The newly evolving therapeutics, cellular therapies as well as vaccines will ultimately be translated into more optimal management of these potentially life-threatening infections in this peculiar group of immunocompromised patients.

\section{REFERENCES}

1. Al-Anazi KA, Al-Jasser AM. Tuberculosis in the era of globalization. Foster City: OMICS Group e Books (2013). Available from: http://www.esciencecentral.org/ ebooks

2. Loto OM, Awowole I. Tuberculosis in pregnancy: a review. J Pregnancy (2012) 2012:379271. doi:10.1155/2012/379271

3. Halse TA, Escuyer VE, Musser KA. Evaluation of a single-tube multiplex real-time PCR for differentiation of members of the Mycobacterium tuberculosis complex in clinical specimens. J Clin Microbiol (2011) 49:2562-7. doi:10.1128/JCM.00467-11

4. Dinnes J, Deeks J, Kunst H, Gibson A, Cummins E, Waugh N, et al. A systemic review of rapid diagnostic tests for the detection of tuberculosis infection. Health Technol Assess (2007) 11(3):1-196.

5. Al-Anazi KA, Al-Jasser AM, Evans DAP. Infections caused by Mycobacterium tuberculosis in patients with hematological disorders and in recipients of hematopoietic stem cell transplantation: a twelve year retrospective study. Ann Clin Microbiol Antimicrob (2007) 6:16. doi:10.1186/1476-0711-6-16

6. Munsiff SS, Nilsen D, Fujiwara PI. Clinical Policies and Guidelines. 4th ed. Bureau of Tuberculosis Control, New York City Department of Health and Mental Hygiene (2008). p. 1-270.

7. Anibarro L, Pena A. Tuberculosis in patients with hematological malignancies. Mediterr J Hematol Oncol (2014) 6(1):e2014026. doi:10.4084/MJHID.2014.026

8. Fanning EA. Globalization of tuberculosis. Can Med Assoc J (1998) 158:611-2.

9. Selgelid MJ. Ethics, tuberculosis and globalization. Public Health Ethics (2008) 1:10-20. doi:10.1093/phe/phn001

10. Oza S. Tuberculosis: an overlooked global threat. Massachusetts Undergrad Res J (2008) 6:25-9. doi:10.1021/jm401735w

11. Tombolyn M, Chiller T, Eisele H, Gress R, Sepkowitz K, Storek J, et al. Guidelines for preventing infections complications among hematopoietic stem cell transplantation recipients: a global perspective. Biol Blood Marrow Transplant (2009) 15:1143-238. doi:10.1016/j.bbmt.2009.06.019

12. Erdstein AA, Daas P, Bradstock KF, Robinson T, Hertzberg MS. Tuberculosis in allogeneic stem cell transplant recipients: still a problem in the 21 st century. Transpl Infect Dis (2004) 6(4):142-6. doi:10.1111/j.1399-3062.2004.00068.x

13. Tomaszewska A, Nasilowska-Adamska B, Prochorec-Sobieszek M, Halaburda K, Szczepinski A, Marianska B. Extrapulmonary tuberculosis following allogeneic stem cell transplantation-a difficult and late diagnosis. Arch Med Sci (2009) 5(3):489-91.

14. Ambrossi GG, Jakubowski A, Feinstein MB, Weinstock DM. Active tuberculosis limited to foreign-born patients after allogeneic hematopoietic stem cell transplant. Bone Marrow Transplant (2005) 36:741-3. doi:10.1038/sj.bmt.1705129

15. Garcia-Elorriaga G, del Rey-Pineda G. Tuberculosis and hematopoietic stem cell transplant: review of a difficult and often underestimated problem. World J Clin Infect Dis (2013) 3(4):70-8. doi:10.5495/wjcid.v3.i4.70

16. Maeda T, Kusumi E, Kami M, Kawabata M, Le Pavoux A, Hara S, et al. Disseminated tuberculosis following reduced-intensity cord blood transplantation for adult patients with hematological diseases. Bone Marrow Transplant (2005) 35:91-7. doi:10.1038/sj.bmt.1704740

17. Al-Anazi KA, Al-Jasser AM. Pulmonary mycobacterial infections in patients with hematological malignancies. In: Azoulay E, editor. Pulmonary Involvement in Patients with Hematological Malignancies. 1st ed. Berlin: Springer-Verlag (2011). p. 269-83.

18. Lee J, Lee MH, Kim WS, Kim K, Park SH, Lee SH, et al. Tuberculosis in hematopoietic stem cell transplant recipients in Korea. Int J Hematol (2004) 79(2):185-8. doi:10.1532/IJH97.A10219

19. de la Camara R, Martino R, Granados E, Rodriguez-Salvanás FJ, Rovira M, Cabrera R, et al. Tuberculosis after hematopoietic stem cell transplantation: incidence, clinical features and outcome. Bone Marrow Transplant (2000) 26:291-8. doi:10.1038/sj.bmt.1702506

20. Yuen KY, Woo PCY. Tuberculosis in blood and marrow transplant recipients. Hematol Oncol (2001) 20(2):51-62. doi:10.1002/hon.681

21. Ramos JF, Batista MV, Costa SF. Tuberculosis in hematopoitic stem cell transplant recipients. Mediterr J Hematol Infect Dis (2013) 5(1):à201306. doi:10.4084/ MJHID.2013.061

22. IP MSM, Yuen KY, Woo PCY, Luk WK, Tsang KWT, Lam WK, et al. Risk factors for pulmonary tuberculosis in bone marrow transplant recipients. Am J Respir Crit Care Med (1998) 158:1173-7.

23. Lee HJ, Kwon JC, Kim SH, Park SH, Choi SM, Lee DG, et al. High Incidence of Tuberculosis among Allogeneic Stem Cell Transplant Recipients in Korea. Poster Abstract session: Transplant and immunocompromised hosts, Abstract number 530 (2012).

24. George B, Mathews V, Srivastava V, Srivastava A, Chandy M. Tuberculosis among allogeneic bone marrow transplant recipients in India. Bone Marrow Transplant (2001) 27:973-5. doi:10.1038/sj.bmt.1702993

25. Martino R, Martinez C, Brunet S, Sureda A, Lopez R, Domingo-Albós A. Tuberculosis in bone marrow transplant recipients: report of two cases and review of the literature. Bone Marrow Transplant (1996) 18(4):809-12.

26. De Assis RA, Kerbauty FR, Rodrigues M, Seguro FS, Aranha LFC, Fayad $\mathrm{L}$, et al. Mycobacterium tuberculosis infection: a rare late complication after cord blood hematopoietic SCT. Bone Marrow Transplant (2008) 43:667-8. doi:10.1038/bmt.2008.379

27. Hughes WT. Mycobacterial infections in bone marrow transplant recipients. Biol Blood Marrow Transplant (2000) 6:359-60. doi:10.1016/S1083-8791(00) 70011-5 
28. Budak-Alpdogan T, Tangún Y, Kalayoglu-Besisk S, Ratip S, Akan H, Baslar $\mathrm{Z}$, et al. The frequency of tuberculosis in adult allogeneic stem cell transplant recipients in Turkey. Biol Blood Marrow Transplant (2000) 6:370-4. doi:10.1016/S1083-8791(00)70013-9

29. Akan H, Arslan O, Akan OA. Tuberculosis in stem cell transplant patients. J Hosp Infect (2006) 62(4):421-6. doi:10.1016/j.jhin.2005.09.020

30. Kindler T, Schindel C, Brass U, Fischer T. Fatal sepsis due to Mycobacterium tuberculosis after allogeneic bone marrow transplantation. Bone Marrow Transplant (2001) 27:217-8. doi:10.1038/sj.bmt.1702737

31. Dolai TK, Kumar R, Bhargava R, Mahapatra M, Mishra P, Seth T, et al. Multiorgan failure due to Mycobacterium tuberculosis and Aspergillus flavas infection after allogeneic bone marrow transplantation. Indian J Hematol Blood Transfus (2008) 24(2):78-80. doi:10.1007/s12288-008-0035-6

32. AlJurf M, Gyger M, Alrajhi A, Sahovic E, Chaudhri N, Musa M, et al. Mycobacterium tuberculosis infection in allogeneic bone marrow transplantation patients. Bone Marrow Transplant (1999) 24:551-4. doi:10.1038/sj.bmt.1701930

33. Russo RL, Dulley FL, Suganuma L, Franca IL, Yasuda MA, Costa SF. Tuberculosis in hematopoietic stem cell transplant patients: case report and review of the literature. Int J Infect Dis (2010) 14(Suppl 3):e187-91. doi:10.1016/j.ijid.2009. 08.001

34. Biral E, Faraci M, Lanino E, Morreale G, Giardino S, Moroni C, et al. Mycobacterium tuberculosis pneumonia and bacteremia after allogeneic hematopoietic stem cell transplant: report of an instructive pediatric case. New Microbiol (2012) 35(3):353-7.

35. Ku S-C, Tang J-L, Hseuh P-R, Luh K-T, Yu C-J, Yang P-C. Pulmonary tuberculosis in allogeneic hematopoietic stem cell transplantation. Bone Marrow Transplant (2001) 27(12):1293-7. doi:10.1038/sj.bmt.1703092

36. Sharma SK, Argawal N, Mukherjee A, Seth T, Mishra P, Xess I, et al. Coexisting pulmonary tuberculosis and mucormycosis in a patient with aplastic anemia post allogeneic stem cell transplantation. Mediterr J Hematol Infect Dis (2011) 3:e2011036. doi:10.4084/MJHID.2011.0036

37. Ullah K, Raza S, Ahmed P, Satti TM, IKram A, Chaudhri QU, et al. Pulmonary tuberculosis in allogeneic stem cell transplant recipients. J Pak Med Assoc (2007) 57(11):567-9.

38. Buyck HCE, Holliman R, Else J, O’Regan L, Willis F, Grubnic S, et al. An usual cause of acute abdomen following allogeneic transplantation: a zoonotic disease revisited. Bone Marrow Transplant (2008) 41:1069-70. doi:10.1038/bmt.2008.29

39. Campos A, Vaz CP, Campilho F, Morais A, Guimaràes MA, Lopes C, et al. Central nervous system (CNS) tuberculosis following allogeneic stem cell transplantation. Bone Marrow Transplant (2000) 25:567-9. doi:10.1038/sj.bmt.1702163

40. Palma J, Catalán P, Mardones P, Santolaya ME. Mycobacterium tuberculosis infection in a pediatric patient who underwent a hematopoietic stem cell transplant. Rev Chilena Infectol (2013) 30(2):202-5. doi:10.4067/S071610182013000200011

41. Shima T, Yoshimoto G, Miyamoto T, Yoshida S, Kamezaki K, Takenaka K, et al. Disseminated tuberculosis following second unrelated cord blood transplantation for acute myeloid leukemia. Transpl Infect Dis (2009) 11(1):75-7. doi:10.1111/j.1399-3062.2008.00354.x

42. Cordonnier C, Martino R, Trabasso P, Held TK, Akan H, Ward MS. Mycobac terial infection: a difficult and late diagnosis in stem cell transplant recipients. Clin Infect Dis (2004) 38:1229-36. doi:10.1086/383307

43. Iguchi T, Yokoyama K, Mitsuishi M, Chen CK, Ikeda Y, Okamoto S. Pulmonary tuberculosis and adenovirus-hemorrhagic cystitis after autologous peripheral blood stem cell transplantation for follicular lymphoma. Rinsho Ketsueki (2005) 46(9):1049-54.

44. Kuan F-C, Lin PY, Hwang C-E, Lu C-H, Chen C-C. Pancytopenia and myeloid maturation arrest in an autologous stem cell transplant recipient. Bone Marrow Transplant (2011) 46:610-1. doi:10.1038/bmt.2010.155

45. Altclas J, Lescano A, Salgueira C, Di Martino A, Brennan V, Campestri R, et al. Multi-drug resistant tuberculosis in bone marrow transplant recipient. Transpl Infect Dis (2005) 7(1):45-46. doi:10.1111/j.1399-3062.2005.00083.x

46. Lee JW, Kwon P-S, Chung N-G, Cho B, Jeong D-C, Kang J-H, et al. Two children with differing outcomes after treatment for pulmonary tuberculosis diagnosed after allogeneic hematopoietic stem cell transplantation. Transpl Infect Dis (2011) 13:520-3. doi:10.1111/j.1399-3062.2011.00641.x

47. Bumbacea D, Arend SM, Eyuboglu F, Fishman JA, Goletti D, Ison MG, et al. The risk of tuberculosis in transplant candidates and recipients: a TBNET consensus statement. Eur Respir J (2012) 40(4):990-1013. doi:10.1183/09031936.00000712
48. Ahmed P, Anwar M, Khan B, Altaf C, Ullah K, Raza S, et al. Role of isoniazid prophylaxis for prevention of tuberculosis in hematopoietic stem cell transplant recipients. J Pak Med Assoc (2005) 55(9):378-81.

49. Lin PL, Flynn JL. Understanding latent tuberculosis: a moving target. J Immunol (2010) 185:15-22. doi:10.4049/jimmunol.0903856

50. Miranda MS, Breiman A, Allain S, Deknuydt F, Altare F. The tuberculosis granuloma: an unsuccessful host defense mechanism providing a safety shelter for the bacteria? Clin Dev Immunol (2012) 2012:139127. doi:10.1155/2012/139127

51. Chensue SW. Chemokines in innate and adaptive granuloma formation. Front Immunol (2013) 4:43. doi:10.3389/fimmu.2013.00043

52. Ufimtseva E. Investigation of functional activity of cells in granulomatous inflammatory lesions from mice with latent tuberculous infection in the new ex vivo model. Clin Dev Immunol (2013) 2013:371249. doi:10.1155/2013/ 371249

53. Kapoor N, Pawar S, Sirakova TD, Deb C, Warren WL, Kolattukudy PE. Human granuloma in vitro model, for TB dormancy and resuscitation. PLoS One (2013) 8(1):e53657. doi:10.1371/journal.pone.0053657

54. Ehlers S, Schaible UE. The granuloma in tuberculosis: dynamics of a hostpathogen collusion. Front Immunol (2013) 3:411. doi:10.3389/fimmu.2013. 00411

55. Boros DL. New perspectives on ancient granulomas. Front Immunol (2013) 4:345. doi:10.3389/fimmu.2013.00345

56. Shaler CR, Horvath CN, Jayanathan M, Xing Z. Within the enemy's camp: contribution of the granuloma to the dissemination, persistence and transmission of Mycobacterium tuberculosis. Front Immunol (2013) 4:30. doi:10.3389/fimmu. 2013.00030

57. Guirado E, Schlensinger LS. Modeling the Mycobacterium tuberculosis granuloma-the critical battlefield in host immunity and disease. Front Immunol (2013) 4:98. doi:10.3389/fimmu.2013.00098

58. Raghuranshi S, Sharma P, Singh S, Kaer LV, Das G. Mycobacterium tuberculosis evades host immunity by recruiting mesenchymal cells. Proc Natl Acad Sci (2010) 107(50):21653-8. doi:10.1073/pnas.1007967107

59. Mittal R. Mesenchymal stem cells: the new players in the pathogenesis of tuberculosis. J Microbial Biochem Technol (2011) 3:3. doi:10.4172/1948-5948. $100000 \mathrm{e} 3$

60. Rai RC, Battacharya D, Das G. Stem cells in infectious diseases. In: Pritti R editor. Insight and Control of Infectious Disease in Global Scenario. InTech (2012). Available from: http://www.intechopen.com/books/insight-and-control-ofinfectious-disease-in-globalscenario/stem-cells-in-infectious-diseases

61. Das B, Kashino SS, Pulu I, Kalita D, Swami V, Yeger H, et al. CD $271^{+}$bone marrow mesenchymal stem cells may provide a niche for dormant Mycobacterium tuberculosis. Sci Transl Med (2013) 5(170):170ra13. doi:10.1126/scitranslmed. 3004912

62. Bhattacharyya R, Shukla M, Nagra S, Banerjee D. Insights from the computational analysis of CD $271^{+}$glycation in mesenchymal stem cells in diabetes mellitus as a predisposition to latent tuberculosis. Bioinformation (2013) 9(16):829-31. doi:10.6026/97320630009829

63. Molicotti P, Bua A, Zanetti S. Cost-effectiveness in the diagnosis of tuberculosis: choices in developing countries. J Infect Dev Ctries (2014) 8(1):024-038. doi:10.3855/jidc.3295

64. Drobniewski F, Nikolayevsky V, Maxeiner H, Balabanova Y, Casali N, Kontsevaya I, et al. Rapid diagnosis of tuberculosis and drug resistance in the industrialized word: clinical and public health benefits and barriers to implementation. BMC Med (2013) 11:190. doi:10.1186/1741-7015-11-190

65. Parsons LM, Somoskövi A, Gutierrez C, Lee E, Paramasivan CN, Abimiku A, et al. Laboratory diagnosis of tuberculosis in resource-poor countries: challenges and opportunities. Clin Microbiol Rev (2011) 24(2):314-50. doi:10.1128/ CMR.00059- 10

66. Palomino JC. Nonconventional and new methods in the diagnosis of tuberculosis: feasibility and applicability in the field. Eur Respir J (2005) 26(2):339-50. doi:10.1183/09031936.05.00050305

67. Vassall A, van Kampen S, Sohn H, Michael JS, John KR, den Boon S, et al. Rapid diagnosis of tuberculosis with the Xpert MTB/RIF assay in high burden countries: a cost-effectiveness analysis. PLoS Med (2011) 8(11):e1001120. doi:10.1371/journal.pmed.1001120

68. Balasingham SV, Davidsen T, Szpinda I, Frye SA, Tønjum T. Molecular diagnostics in tuberculosis: basis and implications for therapy. Mol Diagn Ther (2009) 13(3):137-51. doi:10.2165/01250444-200913030-00001 
69. Tiwari D, Tiwari RP, Chandra R, Bisen PS, Haque S. Efficient ELISA for diagnosis of active tuberculosis employing a cocktail of secretory proteins of Mycobacterium tuberculosis. Folia Biol (Praha) (2014) 60(1):10-20.

70. McNerney R, Maeurer M, Abubakar I, Marais B, Mchugh TD, Ford N, et al. Tuberculosis diagnostics and biomarkers: needs, challenges, recent advances and opportunities. JInfect Dis (2012) 205(Suppl 2):S147-58. doi:10.1093/infdis/ jir860

71. Stolz D, Stulz A, Müller B, Gratwohl A, Tamm M. BAL neutrophils, serum prolactin and C-reactive protein to predict bacterial infection in the immunocompromised host. Chest (2007) 132(2):504-14. doi:10.1378/chest.07-0175

72. Jin M, Khan AI. Procalcitonin: uses in clinical laboratory for the diagnosis of sepsis. LabMedicine (2010) 41(3):173-7.

73. Zhang X, Guo J, Fan S, Li Y, Wei L, Yang X, et al. Screening and identification of six serum microRNAs as novel potential combination biomarkers for pulmonary tuberculosis diagnosis. PLoS One (2013) 8(12):e81076. doi:10.1371/journal.pone.0081076

74. Ruhwald M, Bodmer T, Maier C, Jepsen M, Haaland MB, Eugen-Oslen J, et al. On behalf of TBNET. Evaluating the potential of IP-10 and MCP-2 as biomarkers for the diagnosis of tuberculosis. Eur Respir J (2008) 32(6):1607-15 doi:10.1183/09031936.00055508

75. Qin L-L, Wang Q-R, Wang Q, Yao H, Wen L-T, Wu L-L, et al. T-SPOT.TB for detection of tuberculosis infection among hematological malignancy patients and hematopoietic stem cell transplantation recipients. Asian Pac J Cancer Prev (2013) 14(12):7415-9. doi:10.7314/APJCP.2013.14.12.7415

76. Pai M, Menzies D. Interferon-gamma release assays for diagnosis of latent tuberculosis infection. In: von Reyn CF, Baron EL, editors. UpToDate (2014). Topic: 15352, version: 28, pages: 1-15. Topic last updated: Jul 2014.

77. Lee Y-M, Lee S-O, Choi S-H, Kim YS, Woo JH, Kim D-Y, et al. A prospective longitudinal study evaluating the usefulness of the interferon-gamma releasing assay for producing active tuberculosis in allogeneic hematopoietic stem cell transplantation recipients. J Infect (2014) 69(2):165-73. doi:10.1016/j.jinf.2014. 02.019

78. Demirkazik FB, Akin A, Uzun Ö, Akpinar MG, Ariyürek MO. CT findings in immunocompromised patients with pulmonary infections. Diagn Interv Radiol (2008) 14(2):75-82.

79. Wingard JR, Hiemenz JW, Jantz MA. How I manage pulmonary nodular infiltrates in patients with hematologic malignancies or undergoing hematopoietic cell transplantation. Blood (2012) 120:1791-800. doi:10.1182/blood-2012-02378976

80. Kang M, Deoghuria D, Varma S, Gupta D, Bhatia A, Khandelwal N. Role of HRCT in detection and characterization of pulmonary abnormalities in patients with febrile neutropenia. Lung India (2013) 30(2):124-30. doi:10.4103/0970-2113. 110420

81. Wang Z, Ning X. Clinical diagnosis of bacterial infection via FDG-PET imaging. Can Chem Trans (2013) 1(2):85-104. doi:10.13179/canchemtrans.2013.01.02. 0021

82. Kumar R, Basu S, Torigian D, Anand V, Zhuang H, Alavi A. Role of modern imaging techniques for diagnosis of infection in the era of ${ }^{18}$ f-fluorodeoxyglucose positron emission tomography. Clin Microbiol Rev (2008) 21(1):209-24. doi:10. 1128/CMR.00025-07

83. Harkirat S, Anana SS, Indrajit LK, Dash AK. Pictorial assay: PET/CT in tuberculosis. Indian J Radiol Imaging (2008) 18(2):141-7. doi:10.4103/0971-3026.40299

84. Zulma AI, Gillespie SH, Hoelscher M, Philips PP, Cole ST, Abubaker I, et al. New antituberculosis drugs, regimens and adjunct therapies: needs, advances and future prospects. Lancet Infect Dis (2014) 14(4):327-40. doi:10.1016/S14733099(13)70328-1

85. Okada M, Shirakawa T. Frontier of mycobacterium research-host vs. mycobacterium. Kekkaku (2005) 80(9):613-29.
86. Koul A, Arnoult E, Lounis N, Guillemont J, Andries K. The challenge of new drug discovery for tuberculosis. Nature (2011) 469:483-90. doi:10.1038/nature09657

87. Mase S, Chorba T, Lobue P, Castro K. Provisional CDC guidelines for the use and safety monitoring of bedaquiline fumarate (Sirturo) for the treatment of multi-drug resistant tuberculosis. MMWR Recomm Rep (2013) 62(RR-09):1-12.

88. Grosset JH, Singer TG, Bishai WR. New drugs for the treatment of tuberculosis: hope and reality. Int J Tuberc Lung Dis (2012) 16(8):1005-14. doi:10.5588/ijtld. 12.0277

89. Kwon YS, Jeong BH, Koh WJ. Tuberculosis: clinical trials and new drug regimens. Curr Opin Pulm Med (2014) 20(3):280-6. doi:10.1097/MCP.0000000000000045

90. Lin PL, Dartois V, Johnston PJ, Janssen C, Via L, Goodwin MB, et al. Metronidazole prevents reactivation of latent Mycobacterium tuberculosis infection in macaques. Proc Natl Acad Sci USA (2012) 109(35):14188-93. doi:10.1073/pnas. 1121497109

91. Uhlin M, Andersson J, Zumla A, Maeurer M. Adjunct immunotherapies for tuberculosis. J Infect Dis (2012) 205:S325-34. doi:10.1093/infdis/jis197

92. Erokhin VV, Vasil'eva IA, Konopliannikov AG, Chukanov VI, Tsyb AF, Bagdasarian TR, et al. Systemic transplantation of autologous mesenchymal stem cells of the bone marrow in the treatment of patients with multidrug-resistant pulmonary tuberculosis. Probl Tuberk Bolezn Legk (2008) 10:3-6.

93. Skrahin A, Ahmed RK, Ferrara G, Rane L, Poiret T, Isaikina Y, et al. Autologous mesenchymal stromal cell infusion as adjunct treatment in patients with multidrug and extensively drug-resistant tuberculosis: an open-label phase 1 safety trial. Lancet Respir Med (2014) 2:108-22. doi:10.1016/S2213-2600(13)70234-0

94. Liu J. New vaccine against tuberculosis: current development and future challenges. J Sci Foundation China (2009) 17(2):50-8. doi:10.1088/1005-0841/17/2/ 006

95. Kaufmann SHE. Fact and fiction in tuberculosis vaccine research: 10 years later. Lancet Infect Dis (2011) 11:633-40. doi:10.1016/S1473-3099(11)70146-3

96. Kaufmann SHE, Lambert P-H. New vaccines for tuberculosis. Lancet (2010) 375:2110-9. doi:10.1016/S0140-6736(10)60393-5

97. Kaufmann SHE, Lange C, Rao M, Balaji KN, Lotze M, Schito M, et al. Progress in tuberculosis vaccine development and host-directed therapies - a state of the art review. Lancet Respir Med (2014) 2(4):301-20. doi:10.1016/S2213-2600(14) 70033-5

98. Ottenhoff THM, Kaufmann SHE. Vaccines against tuberculosis: where are we and where we need to go? PLoS Pathog (2012) 8(5):e1002607. doi:10.1371/ journal.ppat.1002607

99. Brennan MJ, Fruth U, Milstein J, Tiernan R, de Andrade Nishioka S, Chocarro $\mathrm{L}$, et al. Development of new tuberculosis vaccines: a global perspective on regulatory issues. PLoS Med (2007) 4(8):e252. doi:10.1371/journal.pmed.0040252

Conflict of Interest Statement: The authors declare that the research was conducted in the absence of any commercial or financial relationships that could be construed as a potential conflict of interest.

Received: 08 May 2014; accepted: 11 August 2014; published online: 26 August 2014. Citation: Al-Anazi KA, Al-Jasser AM and Alsaleh K (2014) Infections caused by Mycobacterium tuberculosis in recipients of hematopoietic stem cell transplantation. Front. Oncol. 4:231. doi: 10.3389/fonc.2014.00231

This article was submitted to Hematology Oncology, a section of the journal Frontiers in Oncology.

Copyright (C) 2014 Al-Anazi, Al-Jasser and Alsaleh. This is an open-access article distributed under the terms of the Creative Commons Attribution License (CC BY). The use, distribution or reproduction in other forums is permitted, provided the original author(s) or licensor are credited and that the original publication in this journal is cited, in accordance with accepted academic practice. No use, distribution or reproduction is permitted which does not comply with these terms. 